\title{
Germanica
}

GERMANICA 18 | 1996

Esthétique théâtrale et représentations du sujet

\section{Matériau M(édée/uller)}

M(edea/üller) - Material

\section{Christian Klein}

\section{(2) OpenEdition}

Journals

Édition électronique

URL : http://journals.openedition.org/germanica/1972

DOI : 10.4000/germanica. 1972

ISSN : 2107-0784

\section{Éditeur}

Université de Lille

\section{Édition imprimée}

Date de publication : 30 juin 1996

Pagination : 115-135

ISBN : 9782098426320

ISSN : 0984-2632

Référence électronique

Christian Klein, « Matériau M(édée/uller) », Germanica [En ligne], 18| 1996, mis en ligne le 13 juin 2013, consulté le 06 octobre 2020. URL : http://journals.openedition.org/germanica/1972 ; DOI : https:// doi.org/10.4000/germanica.1972

Ce document a été généré automatiquement le 6 octobre 2020.

(ㄷ) Tous droits réservés 


\title{
Matériau M(édée/uller)
}

\author{
M(edea/üller) - Material
}

Christian Klein

1 En 1971, Müller insère dans Germania, une pièce sur les événements de juin 1953, un texte en prose intitulé Nocturne (Nachtstück) au statut indéterminé où une instance narrative non identifiable conte sous forme de fable l'histoire d'un « homme », " peutêtre une marionnette", qui court désespérément derrière une bicyclette et bute sur une «barre transversale » visible du seul spectateur chaque fois qu'il s'en approche. L'" homme » retourne les doutes contre lui et se mutile sur scène jusqu'au cri final qui engendre une bouche. Le récit crypté se donne à lire, voir et entendre comme une métaphore qui met conjointement en abyme l'histoire de la RDA et la quête identitaire d'un sujet. On note que ce texte intègre les didascalies dans l'énoncé et pose ainsi au metteur en scène un redoutable problème. En 1972, Müller recourt encore à ce procédé en intercalant entre deux scènes, dans Ciment (1972), des textes en prose, privés de toute indication scénique sur l'énonciateur et les conditions d'énonciation. Ces intermèdes représentent ce que nous appelions des textes-limite ${ }^{1}$. Ils sont, cependant, autant de textes dramatiques en raison de leurs conditions d'insertion, voire par simple acte définitoire de l'auteur. Ainsi Paysage sous surveillance, récit en prose sans personnage ni locuteur, qui intègre les didascalies dans le récit, a été écrit pour le festival de théatre de Graz. Même si des séquences comme le Texte de l'Hydre ou la Libération de Prométhée, ont fait par la suite l'objet de publications séparées, ils sont et restent des fragments dramatiques qui posent tous la question du statut du sujet et de sa problématique émancipation.

2 Un autre texte, Rivage à l'Abandon Matériau Médée Paysage avec Argonautes, présente les mêmes caractéristiques, et a curieusement été négligé jusqu'à ce jour par la recherche ${ }^{2}$. Le texte théâtral se présente comme un ensemble où trois énoncés se succèdent selon un principe de juxtaposition qui fait écho à rénumération énigmatique du titre. La première et la troisième parties (Rivage à l'abandon / Paysage avec Argonautes) n'ont pas d'instance énonciatrice identifiable. Rivage à Vabandon renvoie à un «auteur impersonnel $»^{3}$. Le «je» qui surgit, vers 10 , au milieu de ce qui semble être un monologue intérieur, intervient dans des séquences à forte intonation (en lettres 
majuscules). Celles-ci se lisent comme l'irruption de fragments de conversation d'origine inconnue, sans indication scénique ou verbale qui permettrait d'identifier son auteur ni les conditions de cette énonciation. Les pronoms personnel, «mich», possessif, «mein», s'intercalent entre un «ich» et un « du » qui, certes, introduisent un conflit à trois entre un homme et deux femmes en terme de liaison amoureuse, de séduction et de trahison, mais sont privés de toute référence à un lieu, un temps, un contexte - sauf celui du récit en vers libres qui associe par simple linéarité une succession de situations dont le lecteur (avant toute mise en scène) traque un possible dénominateur commun.

3 La troisième partie, Paysage avec Argonautes, est un récit à la première personne : on peut l'attribuer à un homme, à Jason lui-même, mais en tant que représentant des hommes qui ont fondé la civilisation moderne et ses productions annexes ou périphériques, les déchets du "progrès ", un moi masculin donc qui se définit par sa diffraction et sa décomposition.

4 Au centre de cette trilogie, Matériau Médée installe un texte dramatique avec trois personnages, Médée, la Nourrice et Jason. Les enfants de Médée sont interpellés, pris à partie et assassinés par Médée, selon la version d'Euripide, au cours de la scène, sans qu'il soit possible de déterminer leur présence, si ce n'est par inférence à partir du récit de Médée.

5 Aucune indication scénique ne décrit, ni ne précise les entrées et sorties des personnages, ni leurs gestes, ni leurs déplacements.

6 Ce montage de trois textes est le produit d'une écriture elle-même éclatée dans le temps, puisque Müller écrivit le premier texte, «à quelques lignes près », en 1952, à un moment où il cherche une écriture et expérimente encore entre les genres littéraires, le second pour moitié vers 1967 , le troisième en $1982^{4}$. Ce travail de sédimentation, de greffe et d'imbrication par intervalle couvre trente années d'écriture et d'expériences dramatiques continues dont il porte les fruits selon un principe de maturation particulier. Il en résulte, ce sera notre première hypothèse, que l'absence de didascalies, loin d'être accidentelle ou anecdotique, est liée au projet, c'est-à-dire à une stratégie consciente, et à la crise du sujet mullérien telle qu'elle se développe au fil de son œuvre dramatique, depuis Mauser jusqu'à Paysage sous surveillance.

7 Nous aborderons ici cette question en interrogeant successivement chaque moment de la trilogie avec, comme perspective, de préciser le statut dramatique de ce singulier tryptique.

\section{Paysage à l'abandon}

Le premier volet commence par la description d'un paysage :

See bei Straußberg Verkommenes Ufer Spur

Flachsinniger Argonauten

Schilfborsten Totes Geäst

[...] Fischleichen

Glänzen im Schlamm Keksschachteln Kothaufen TROMMS ACT CASINO

Die zerrissenen Monatsbinden Das Blut

Der Weiber von Kolchis (M 7, 91)

9 Si l'on excepte le vers 4 qui infère la confidence d'une voix intérieure ${ }^{5}$, la séquence peut parfaitement se lire comme une didascalie, à condition toutefois de ne pas situer 
Straußberg en Prusse orientale, près de Francfort sur l'Oder, à la frontière entre la RDA et la Pologne, et d'accepter l'anachronisme qui réunit l'univers référentiel du mythe de la toison d'or (les Argonautes et les femmes de Colchide, royaume de Médée) et les détritus de la société de consommation moderne, avec les préservatifs, marque fromms act, et les mégots de cigarettes de RDA, marque Casino. Le lieu de ce que nous attendons être une action unit deux cultures extrêmement éloignées dans le temps et l'espace. La description incombe à un auteur qui accompagne le texte dramatique, mais qui s'en distingue fondamentalement. Cependant, outre l'anachronisme, l'insertion du vers 4 et les articles définis «Die zerrissenen Monatsbinden Das Blut/Der Weiber von Kolchis» transgressent le simple énoncé d'une indication scénique. Le texte devient un parcours polyphonique dans lequel la présence subjective de l'auteur disparait au profit de la voix de plusieurs autres, ce qui définit « un trait distinctif» (A. Ubersfeld) de l'écriture théâtrale. Pour autant, le texte intègre une série de discours d'origines les plus diverses, entre le descriptif, l'énonciatif, le constatif, le subjectif confidentiel («Traum von einem ungeheuren Beischlaf»), etc. sans qu'un hypothétique interlocuteur vienne contribuer à identifier, dans chaque nouvelle situation, celui qui parle.

La mer Égée et la mer de Marmara, où les héros du grand poème épique d'Apollonios de Rhodes multiplièrent les escales et les aventures, où ils affrontèrent de violentes tempêtes et les remous du Bosphore, où ils échappèrent aux rochers mouvants pour atteindre enfin, aux pieds du Caucase, la Colchide où Jason triomphera des guerriers géants nés des dents du dragon et s'emparera de la Toison d'or avec la complicité de la magicienne Médée, est devenu un petit lac de Prusse orientale. La synecdote réduit l'épopée à l'anecdote. Tout à la fois elle infère un lieu mythique et le réduit à un simple espace à l'abandon. La référence au mythe met en scène un lieu d'arrivée qui en signifie le déclin, ou plutôt qui substitue une image désillusionnée à l'aura d'une œuvre poétique qui s'avère avoir été mystificatrice. Müller évoque la fin peu glorieuse de Jason écrasé par la poupe vermoulue de son propre bateau :

Bis ihm die Argo den Schädel zertrümmert das nicht mehr gebrauchte Schiff

Das im Baum hängt Hangar und Kotzplatz der Geier im Wartestand

11 La disproportion entre le rêve thessalien et la réalité prussienne est liée à l'exaltation de l'enfance (d'une civilisation) et la mesure du regard adulte. Le retour au point de départ par le détour de l'ellipse nous fait prendre la mesure du chemin parcouru. Dans un poème, écrit lui aussi au début des années cinquante, Müller pose la question de cet écart entre les projets d'avenir et le présent qu'il est devenu :

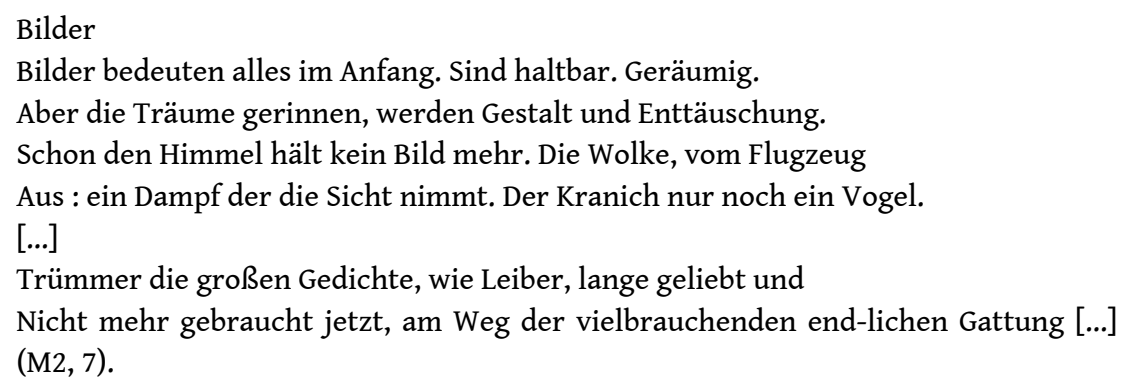

12 L'auteur dispose des « ruines des grands poèmes » comme matériau poétique. L'espèce humaine qui consomme beaucoup n'en a plus usage, sauf, propose Müller près de trente ans plus tard, pour mettre en perspective le prix des "progrès» de la Civilisation occidentale. 
Le mythe posait le voyage comme le creuset de l'expérience (Er-fahrung). Müller transpose ce thème, fondateur de culture, dans les banlieues urbaines du XXe siècle : les Argonautes d'hier sont aujourd'hui des travailleurs (immigrés) fatigués qui se déplacent dans des trains bondés pour se rendre au travail, le sexe en éveil face aux femmes fardées qu'ils croisent sur leur chemin, dans les compartiments. Müller transpose le désir de conquête et substitue la grisaille des métropoles modernes à la griserie des grands espaces. Ce faisant, il intrique la conquête territoriale et la conquête masculine. Tous deux participent d'un même désir de soumettre l'autre. Dans ce contexte de remise à jour des représentations, la mobilité devient un travail.

En quelques énoncés fragmentés Müller met en place des repères sybillins sur le statut de la femme : après l'évocation d'une scène d'orgasme, dont on ignore quels en sont les partenaires, une femme délaissée prend sa rivale à partie et tente de séduire de nouveau le compagnon infidèle. Ces lambeaux de conversation définissent la femme comme objet du désir masculin, dans un rapport de dépendance affective et sociale vis à vis de l'homme, voire dans un rapport de prostitution (STOSS MICH KOMM SÜSSER). La scène pérennise une situation archaïque. Elle condense, dans sa version actualisée, le point de départ, en marge du mythe, de la pièce d'Euripide, que l'on présente par ailleurs comme le "fossoyeur" de la tragédie grecque: Médée accuse Jason de l'abandonner, après avoir tout obtenu d'elle, pour épouser la fille du roi Créon. Car au fonds de cette ère de conquête, comme au fonds des mers Egée et d'Armara ou au fonds du lac de Straußberg, il y a le personnage complexe de Médée, esclave hier, révoltée sur la scène du théâtre aujourd'hui :

Auf dem Grund aber Medea...

L'auteur entreprend de « déterrer » les mort(e)s et « de les montrer au grand jour ${ }^{6}$.

L'absence de ponctuation, même si les majuscules signalent ici et là le début d'une nouvelle proposition au milieu d'un vers, et surtout l'absence de didascalies posent la continuité, tant historique que narrative - l'une exposant l'autre - comme principe de lecture des temps modernes. Elles posent aussi la substitution comme principe d'équivalence, elles posent enfin la différence entre le passé et le présent selon un jeu de spirale qui tend à superposer un plan actualisé et désillusionné sur un plan mythique tout en assurant la reconnaissance d'un écart signifiant.

Dans un dernier enchaînement, Müller juxtapose deux arrêts-image d'une violence extrême: le premier évoque les exécutions pour l'exemple de soldats allemands qui refusèrent de participer aux actes de guerre. Il s'agit ici d'une citation d'un texte de Müller, Tracteur :

EINIGE HINGEN AN LICHTMASTEN ZUNGE HERAUS VOR DEM BAUCH DAS SCHILD ICH BIN EIN FEIGLING (M2, 9)

Dans Tracteur, un texte commencé aussi au début des années cinquante (1955/1961), un soldat refuse de miner un champ de pommes de terre pendant la retraite après la défaite de Stalingrad, en pensant au paysan russe qui en aura besoin après la guerre. Il est pendu pour haute trahison. Le soldat, lui-même un paysan déraciné par un expansionnisme destructeur, pense à la survie de l'autre - ici le paysan russe - comme les déserteurs du paratexte qui commente la scène fuyaient la guerre pour survivre. Or, fait remarquer Müller, on trahit toujours pour survivre ${ }^{7}$. En d'autres termes, survivre, c'est, quelque part, trahir. Le bloc de deux vers puise dans l'imaginaire collectif ces images d'exécutions sommaires qui ont été depuis condamnées par les vainqueurs. À la trahison d'ordre privé se superpose la trahison d'une communauté nationale. Le propos 
de Müller ne se laisse pas déchiffrer totalement. La référence à une histoire récente donne au texte une forte charge émotionnelle que toute didascalie remettrait en cause et qui implique irrésistiblement le spectateur allemand. Elle assure le lien entre le récit théâtral, le mythe et la situation du destinataire au moment de la lecture ou de la représentation. Cette (ef)fusion renforce le passage de la Prusse du $\mathrm{XX}^{\mathrm{e}}$ siècle à la Colchide, plusieurs siècles avant notre ère, engagé par le vers 1 et conditionne notre regard sur la tragédie de Médée et de Jason, un regard qui porte déjà l'expérience des travailleurs immigrés en Europe et des pendaisons pendant la débâcle allemande de 1944.

19 La répression féroce du désir de survivre, au nom d'une conquête militaire hitlérienne mise par ailleurs en échec, suscite encore l'horreur aujourd'hui. Elle se heurte au plan suivant qui évoque Médée, le corps morcelé de son frère Apsyrtos dans les bras, tué lors d'une embuscade qu'elle lui tendit elle-même pour échapper aux Colques lancés à la poursuite de Jason par son propre père. Le cadavre du frère, coupé aux extrémités selon un rite apotropaïque, accuse Médée de trahison envers sa famille et son peuple, par amour pour Jason et pour le succès d'une expédition qui pose plus de problèmes qu'elle n'a semblé en résoudre. Il accuse aussi, par contamination, le désir naturel de survivre qui a poussé Médée à trahir. En fait, ce désir - que le plan précédent vient de légitimer contre ceux qui le condamnent - est reconnu et la révolte identitaire de Médée dans le texte suivant donnera la parole non à une criminelle, mais à une victime.

20 La succession de ces plans transforme le texte en métaphore plus large où chaque élément est convoqué pour actualiser une partie de ses signifiés. Le topos de la trahison ainsi constitué est débarrassé du discours moralisateur, qui l'accompagne habituellement pour légitimer la répression de l'acte.

21 Le lecteur ne parvient pas à rationaliser totalement ce topos, mais il perçoit confusément un lien organique entre les deux situations, sur le modèle du prologue de Brecht à Antigone et de la pièce de Sophocle. Il est déstabilisé et, de ce fait, disponible pour une relecture du mythe. À quelqu'un qui objectait qu'on ne comprenait pas une de ses pièces, Müller soulignait la productivité de cette résistance du sens :

Nur so aber wird es Erfahrung, mit der man länger umgeht ${ }^{8}$.

\section{Matériau Médée (vers 34-222)}

22 Au soliloque polyphonique succède un dialogue, Matériau Médée, qui réunit Médée, la nourrice et Jason. La scène s'inspire de la pièce d'Euripide dont elle réduit le nombre des personnages. Elle commence par un dialogue entre Médée et la nourrice, puis entre Médée et Jason, se poursuit en alternant une interpellation des enfants, sur la présence desquels aucune indication scénique ne permet de statuer, et de Jason qui, si Ton suit les propos de Médée, a dû entrer au vers 53, et devrait s'être absenté au plus tard à la fin du vers 157 pour porter le funeste cadeau de Médée à sa nouvelle fiancée. Il revient au vers 221. Matériau Médée peut aussi se lire, à partir du vers 70 jusqu'au vers 220, comme un long monologue de Médée sans aucun des interlocuteurs cités dans ses propos.

23 Matériau Médée pose de façon centrale la question de l'identité. La scène introduit Médée comme la femme de Jason

Medea : Jason Mein Erstes und mein Letztes Amme

Wo ist mein Mann 

magicienne qui prenait en charge les problèmes de Jason et volait la Toison d'or. L'adjuvante se cherche comme sujet. La confrontation avec l'image de ce qu'elle est devenue introduit une disjonction et une révolte du personnage. Ici aussi, aucune didascalie ne signale de rupture de ton. Celle-ci est cependant rendue manifeste par la réaction de Jason. Faut-il alors la jouer, ou laisser le spectateur la reconstituer rétrospectivement?

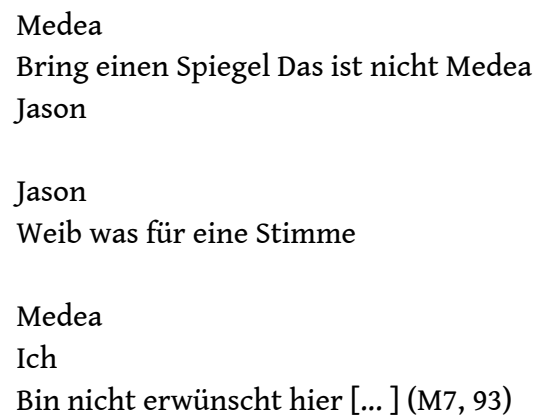

La typographie tient lieu en partie d'indication scénique : la majuscule au milieu du premier vers suggère une pause, un examen; la question de Jason transforme l'énoncé précédent de son nom en une sorte de cri ou la manifestation d'un effroi qui serait proféré par sa compagne devant l'abîme de cette révélation soudaine entre deux identités concurrentes, entre l'ancienne et l'actuelle ; l'absence de point d'interrogation peut s'interpréter comme une confirmation du changement qui s'opère et devant lequel Jason est a priori désarmé, elle relève plus du constat que du dialogue. Enfin, le pronom personnel mis en exergue (Ich) est l'indice de l'émergence d'une parole, dont l'émetteur n'est encore qu'un sujet grammatical - où s'énonce le reproche d'un sujetobjet à un partenaire dont la qualité de sujet, dans un mouvement inverse, va être remise en cause, puis niée pendant le monologue qui suit, avant d'être diffractée et déconstruite avec le troisième volet, Paysage avec Argonautes. L'étonnement de Jason (qui vient d'arriver?) porte précisément sur «la voix » de Médée. Un ton nouveau, comme une déchirure, annonce une prise de conscience et une rébellion qui explose dans le « monologue » de Médée (à partir du vers 71).

La prise de parole par une Médée double est précédée et préparée par un échange sybillin en trois temps qui installe cette situation de rupture. Premier échange,

Jason : Wann hört das auf

Medea : Wann hat es angefangen

Au delà de cette répartie vive, face à un Jason qui entend couper court aux plaintes de son épouse, l'article défini «das» renvoie, à l'insu de celui qui énonce ces mots, au 
destin collectif des femmes de Colchide, lequel prend valeur paradigmatique en raison du lieu scénique où elles sont proférées. L'article défini infère tout ce que Rivage à Vabandon a mis en place dans l'imaginaire du lecteur/spectateur sur le déclin d'une civilisation qui programmait déjà, elle aussi à son insu, la production de ses « déchets ", sa propre agonie lente comme prix de ses victoires. En retournant la question, Médée/ Miiller modifie les termes du débat et impose un retour à l'origine.

Deuxième échange :

Jason : Was warst du vor mir Weib

Medea : Medea

La question de Jason, en raison même de l'absence de didascalie et de point d'interrogation, sous-entend une réponse : «rien », que la réplique de Médée anéantit aussitôt : avant de rencontrer Jason, Médée avait un nom, des qualités, un passé. Le dialogue remet en suspens le récit des aventures communes et engage un retour au point de départ, au point de dérive ou de rupture, à savoir la rencontre entre deux cultures, le moment où l'une, celle, masculine, de l'épopée, instrumentalise l'autre. En quelques vers, Médée est passée de l'épouse colonisée et sacrifiée à la Barbare qui revendique son identité comme sujet.

Il en résulte que le dialogue avec la nourrice - qui porte en elle toute l'expérience des femmes de Colchide (et d'ailleurs) - puis avec Jason n'a de fonction que transitoire. Il installe le monologue de Médée.

34 Ce monologue est lancé par un troisième échange :

Medea : Du bist mir einen Bruder schuldig Jason

Jason : Zwei Söhne gab ich dir für einen Bruder

Le monologue se construit sur cette "équivalence " entre un frère et deux fils : le sacrifice des fils, nés de l'union avec Jason, c'est-à-dire de la conquête de Médée par l'homme colonisateur, doit annuler ladite colonisation de la femme et de la Colchide. Le leitmotiv du retour des fils à leur " propriétaire " (vers 71-72,115-116), sous la forme de leur disparition ${ }^{9}$, métaphorise la maternité, et au delà la fécondité de la femme, comme ce qui signifie l'histoire moderne : par son alliance et son union avec Jason, Médée a contribué à enfanter la civilisation européenne. De sorte que sa révolte prend en charge celle de toutes les victimes des colonialismes : elle prolonge la révolte d'Ophélie dans Hamletmaschine, elle passe à l'acte. Elle tue en elle cette maternité qui la lie au conquérant et mène la reconquête de la parole à son terme.

Quittant le «dialogue » pour le "monologue », on passe de l'autodestruction de Médée comme consécration sacrificielle de l'esclavage de la fille de Colchide (vers 64 : «Willst du mein Blut trinken Jason») à l'autodestruction de l'esclave en Médée au nom de l'émancipation à accomplir. Le texte de Müller propose trois personnages de femme. La nourrice est témoin vivant de la pérennité de l'esclavage des femmes (vers 48-49). Creuse est définie comme un double de Médée : elle est fille de roi, comme Médée, elle est jeune et désirable, comme le fut Médée. Médée se désigne comme « la chienne » et la «putain» de Jason (vers 76, 109), et désigne sa rivale dans les mêmes termes (vers $100,125)$. Creuse doit reprendre ses enfants à elle dans son propre giron encore virginal. Enfin, elle lui offre - ici Müller innove sur Euripide - sa propre robe de mariée. En assassinant Creuse, elle assassine en effigie la princesse naïve qu'elle a été et met fin ainsi au cycle infernal que Jason s'apprête à relancer. La fille de Créon meurt en éprouvant les souffrances que Médée a endurées. Elle se met littéralement dans sa peau. Le déplacement symbolique signe le théâtre comme lieu de représentation 
visuelle et verbale, la mise en espace et la projection des fantasmes de Médée - et probablement d'une partie des spectateurs/trices. La mort de Creuse, en lieu et place de Médée, incarne le moment cathartique par excellence.

Le soliloque de Médée consacre la mort du partenaire dramatique. Le moment où l'éternel(le) adjuvant(e) s'affirme comme sujet par la conquête de la parole est aussi le moment où le « héros » masculin du récit épique, Jason, perd son identité, symbolisée ici par la perte de son nom, à la fin du monologue du moi féminin :

Medea : Ich bin Medea Ich

Habt ihr kein Blut mehr Jetzt ist alles still

Die Schreie von Kolchis auch verstummt Und nichts mehr

Jason : Medea

Medea : Amme Kennst du diesen Mann (M7, 98, vers 218-222)

La fin de Matériau Médée consacre la destruction de l'autre comme interlocuteur. L'interlocution se fond dans un récit à la première personne. Le second volet du tryptique, où dominent en apparence un dialogue et une histoire, consacre en réalité la mort du dialogue, le marginalise, le déplace à la périphérie. À ce titre l'absence de didascalie en est une : elle invite à supprimer les entrées et les sorties, non seulement parce que leur réinsertion n'aurait aucune valeur sémantique (car trop évidente), mais aussi et surtout parce que un personnage "secondaire", la magicienne Médée, qui assurait jusque là le succès des épreuves du héros et donc la poursuite de l'histoire, s'installe au centre, mieux, prend en charge la totalité du récit, et ce de façon tyrannique et brutale, sans aucune concession, ni partage du pouvoir.

Les deux actions criminelles, que Matériau Médée met en scène, ne sont l'objet d'aucune indication scénique qui en préciserait les conditions dramaturgiques.

Le récit de la mort de Creuse est intégré dans le monologue de Médée (vers 158-186). Il s'ouvre et se ferme sous le signe d'un spectacle que Médée offrirait à ses enfants :

Seht eure Mutter gibt euch jetzt ein Schauspiel

Wollt ihr sie brennen sehn die neue Braut (vers 158-159)

[...]

Sie brennt Lacht ihr Ich will euch lachen sehn

Mein Schauspiel ist eine Komödie (vers 185-186)

41 La reprise anaphorique de l'embrayeur « maintenant » (jetzt: vers 158 - vers 172, 173, $176,177,182)$ ponctue le passage entre l'annonce («voulez-vous la voir brûler » et l'action au présent: «elle brûle »). Dans la tradition théâtrale, la teichoscopie fait décrire ce qui se passe en coulisse dans l'instant même où l'observateur en fait le récit. Il s'agit d'éviter, par cette convention, de représenter une scène violente, où techniquement difficile à jouer, en donnant au spectateur l'illusion qu'elle se passe réellement. Müller recourt à ce procédé dans Hamletmaschine en transposant un inceste entre Hamlet et sa mère au plan verbal et symbolique ${ }^{10}$. Ici encore, Müller détourne cette technique. L'embrayeur « maintenant» donne au récit un indice d'existence et de réalité tout en désignant, de façon paradoxale, sa dimension hallucinatoire. Le récit d'un fantasme privé devient, en raison de son énonciation dans un lieu public, de la présence physique de l'actrice et des spectateurs, une représentation et une expérience collectives. La scène est réactualisée dans sa version symbolique, là où des indications scéniques la relégueraient dans un passé mythique. Le déplacement magique, dans la mesure où la femme de Jason meurt en la personne de Creuse pour libérer la magicienne de Colchide, s'opère d'autant mieux qu'aucune didascalie ne vient troubler 
ce dédoublement et ce transfert. La superposition Creuse/Médée s'invente à l'intérieur du monologue.

De même l'assassinat des enfants gagne d'autant plus en signification symbolique que leur présence reste strictement verbale ou synecdotique: dans la mise en scène de Bochum, les deux enfants sont représentés par deux boites de conserve que l'actrice écrase pendant son discours. Traces vivantes du récit sur l'expédition qui rapporta la Toison d'or, ils sont tout à la fois enjeu symbolique du conflit qui oppose le père (incarnation d'une race d'explorateurs modernes), et la mère (paradigme du refus de cette histoire), interlocuteurs invisibles de Médée, spectateurs de la mort de Creuse et acteurs de leur propre mort. Médée assure seule la régie totale de leur présence, de leurs réactions imaginaires et de leur disparition criminelle. Cette absence didascalique élimine tout sentimentalisme intempestif et centre la scène sur le discours d'une révolte libératrice. Le paradigme Médée ainsi construit gagne en épure et en lisibilité. Il s'actualise sans résistance: Médée, indique l'auteur en annexe, peut se jouer indifféremment "au bord d'un lac près de Straußberg", ou au bord d'une "piscine envasée à Beverly Hills» ou encore dans la «salle de bains d'une clinique psychiatrique " (M 7, 101). Le discours de Médée se lit comme un délire qui dit une situation à son point d'arrivée, tout en précisant les causes historiques et idéologiques et le prix monstrueux de la libération. Le dépouillement de la dramaturgie installe un lieu où une femme anonyme peut se prendre pour Médée et parler de sa situation à partir du mythe. Ces actualisations sont envisagées par l'auteur, comme le montre l'entretien avec des journalistes du Spiegel. Devant les réticences de Müller à commenter son propre texte, un journaliste propose une lecture par équivalences :

Question: Médée est-elle une citoyenne de la RDA qui se laisse attirer à l'Ouest par son amant ? Est-elle une Tchèque qui, en 1968, se commet avec un occupant russe? Est-elle une Vietnamienne qui sort avec un Yankee?

Müller répond :

Müller : Ça fait pas mal de bonnes interprétations.

Question : Vous voudriez que Médée soit tout cela en même temps?

Müller : Elle peut être aussi bien une Turque en RFA. Tout ce que vous voudrez ${ }^{11}$.

La variété des transpositions possibles n'épuise pas le champ des interprétations. Müller efface de ses textes, ici plus encore, les éléments référentiels qui limiteraient la productivité du texte dramatique. Dans cette démarche consciente, Müller se range du côté de Kafka contre Brecht :

Parmi les sujets discutés à Svendborg entre Brecht et Benjamin, il y a celui de Kafka. Entre les lignes de Benjamin se lit la question de savoir si la parabole kafkaïenne n'offre pas davantage d'espace, n'est pas capable d'absorber (et de rendre) davantage de réalité que la parabole brechtienne. Et cela, non pas bien que mais parce qu'elle décrit/donne à voir des "gestes " sans système référentiels, non orientés vers un mouvement (praxis), non réductibles à une signification, étranges plutôt que produisant de l'étrangeté, sans morale ${ }^{12}$.

L'auteur renonce à la place conventionnelle que prévoit l'écriture dramatique pour mieux développer l'expression des exclus de l'histoire. Une anecdote connue illustre cette attitude de Müller dans son travail d'auteur dramatique pendant les années quatre-vingt. Montant à la tribune, parmi nombre d'intellectuels portés par la chute du mur et l'agonie de la RDA, le 4 novembre 1989 à Berlin, Müller se contenta de lire un tract qui appelait à fonder des syndicats libres. L'écrivain s'effaçait ainsi devant un groupe de citoyens privés pendant longtemps du moyen de s'exprimer par eux-mêmes 
et leur donnait simplement la parole. Comme Maïakovski arrivant « dans l'arène de la lutte des classes » avec son célèbre poème « 150 Millions » qui portait, ajoute Müller, « le nom de son auteur $»^{13}$, Müller supprime la voix de l'auteur du texte dramatique en supprimant les didascalies dans Rivage à l'abandon Matériau Médée Paysage avec Argonautes.

On mesure le chemin parcouru depuis Hamletmaschine où une didascalie indiquait au metteur en scène et au lecteur/spectateur que le personnage-titre, qui parle à la première personne, procède sur scène «à la mise en pièces de la photographie de l'auteur $»^{14}$. Dans Hamletmaschine, Müller met en crise l'idéalisme d'une philosophie occidentale du sujet et soumet l'intellectuel européen à une autocritique masochiste. Mais il ne s'agit pas alors de représenter un quelconque décentrement du sujet, sur le modèle des théories postmodernistes. La surenchère du "héros " dans ses propos autoaccusateurs et l'indication scénique citée plus haut signalaient encore une présence de l'auteur (même si c'était pour inviter à déchirer la photo de l'auteur) ${ }^{15}$. Le recours à une indication scénique, dans Hamletmaschine, contredit le geste prescrit et en indique les limites : il s'agit bien d'une action spectaculaire, d'un exhibitionisme qui traduit un désespoir.

Cette fois l'auteur disparaît comme intermédiaire entre les spectateurs et la représentation, il gomme la voix subjective qui situe le texte dramatique, qui précise le cadre, les conditions de renonciation. En agissant ainsi, il abandonne le futur spectateur au tête à tête avec une dramaturgie qui le force à « regarder l'histoire dans le blanc des yeux $»^{16}$. Cette histoire est tout autant le récit mythique dans sa version contemporaine que l'Histoire des hommes. L'un et l'autre tendent à se confondre au cours de la représentation. Une telle confusion programmée des temps « pourrait être la fin de la politique et le commencement d'une histoire de l'homme $»^{17}$.

\section{Paysage avec Argonautes}

48 Le troisième volet donne la parole à un moi masculin que l'on peut identifier à Jason. Le « il » des grands récits s'exprime à la première personne. Il n'est pas une instance narrative qui proclamerait ses exploits à la face du monde, mais un être qui a perdu la cohésion du sujet cartésien :

Soll ich von mir reden Ich wer

Von wem ist die Rede wenn

Von mir die Rede geht Ich Wer ist das (M7, 98, vers 223-225)

Curieusement, le moi masculin parle de lui à partir de ce que disent les autres. Son identité résulte de la somme des discours sur lui. Il est la somme des aventures qu'on lui prête, le croisement de récits :

Ich Wer ist das

Im Regen aus Vogelkot Im Kalkfell

Oder anders Ich eine Fahne ein

Blutiger Fetzen ausgehängt Ein Flattern

Zwischen Nichts und Niemand [...]

Ich meine Seefahrt

Ich meine Landnahme (ibid. vers 225-237) 
masculin n'existe que par le récits de ses conquêtes, de ses explorations. Il est itinérant. Il est le "héros » qui va d'aventures en aventures. Ses déplacements le définissent. Il meurt effectivement après avoir ramené la Toison d'or :

Müller : Sa mort indique le seuil, le passage du mythe à l'histoire : Jason est écrasé par son navire ${ }^{18}$.

51 Ici nul besoin d'indications scéniques, car l'auteur prive le «personnage » de tous les artifices qui le façonnent comme héros des temps modernes et père de tous les explorateurs. "Jason" n'organise pas son discours, il juxtapose des séquences qui mettent au jour un désarroi, une errance parmi les "ruines» du $\mathrm{XX}^{\mathrm{e}}$ siècle, les charniers et les décombres des guerres modernes, mais aussi les terrains vagues des cités-dortoir de notre civilisation urbaine. Toute intervention de l'auteur fonctionnerait automatiquement, à l'insu même de l'auteur, comme une organisation du sens par ceux-là mêmes qui écrivent l'histoire des hommes depuis Apollonios de Rhodes jusqu'à aujourd'hui. En d'autres termes, le discours de «Jason » est écrit à la périphérie du sujet occidental, à la périphérie de l'eurocentrisme. Sur ce point, Müller rejoint la critique qui monte alors en RDA contre la philosophie des lumières et l'optimisme du progrès, et mêle sa voix à celle de Benjamin, de Christa Wolf etc. Le représentant du moi masculin ne trouve pas la signification de ses actes dans une histoire linéaire. Il se présente à la fois comme prédateur et comme victime de l'Histoire. Son errance, considérée par convention comme fondatrice de sens, ne permet pas de fixer un sens à l'histoire. Ce sens est toujours différé dans la mesure où il n'est pas fondé sur un seul terme, mais sur un système mouvant de significations.

À ce titre, on peut estimer que la dépossession de son identité légendaire revient à une sorte d'expropriation du moi masculin, soumis aux multiples accidents qui le menacent d'anéantissement :

Ich Mein Tod

Im Regen aus Vogelkot Im Kalkfell

Der Anker ist die letzte Nabelschnur

Mit dem Horizont vergeht das Gedächtnis der Küste

Vögel sind ein Abschied Sind ein Wiedersehn

[...]

Dünn zwischen Ich und Nichtmehr Ich die Schiffswand (M7, 98, vers 238-244)

Exposé aux vents et aux tempêtes d'une nature hostile, il est un petit d'homme arraché au giron maternel. Le patriarcat évoqué dans le premier texte trouve ici le démenti d'une puissance qui se fonde sur le déracinement et l'angoisse. L'agressivité des conquêtes est généré par une fragilité constitutive. de métaphores, de confidences, de citations juxtaposées. La présence du sens devient irréalisable dans la mesure où chaque image renvoie à des significations antérieures ou postérieures. La postmodernité de ce récit déconstruit tient à ce que Derrida appelle la différence :

La différance, c'est ce qui fait que le mouvement de la signification n'est possible que si chaque élément dit « présent » [...] se rapporte à autre chose que lui-même, gardant en lui la marque de l'élément passé et se laissant déjà creuser par la marque de son rapport à l'élément futur [...] $]^{19}$.

Le moi masculin a perdu sa (fausse) cohésion. À l'inverse des didascalies qui deviennent des textes de théâtre (songeons à Kaspar de Handke ou à Concert à la carte de Kroetz), leur élimination résulte de la volonté de Müller d'inscrire dans le texte dramatique le 
lieu d'où il parle, c'est-à-dire les ruines d'une civilisation fondée sur la conquête de l'autre et la production de "déchets» qui sont autant de traces du prix de cette histoire.

\section{NOTES}

1. Sur cette question Christian Klein: "De la prose au théâtre jusqu'aux textes-limite, ou pourquoi n'écrivez-vous pas de la prose, Monsieur Müller?», in : M.-Ch. Autant-Mathieu (sous la dir.) : Écrire pour le théâtre, éd. CNRS, Paris, 1995, p. 45-66.

2. Signalons cependant Klaus Teichmann : «Der verwundete Körper. Zu Texten Heiner Müllers», Burg-Verlag, Freiburg/B, 1986, p. 197-211 et surtout l'étude de Bettina Gruber : Mythen in den Dramen Heiner Müllers. Zu ihrem Funktionswandel in den Jahren 1958 bis 1982, éd. Die Blaue Eule, Essen, 1989, p. 116-164.

3. Nous empruntons cette définition du didascalos à Milagros Ezquerro et al. Manual de anâlisis textual, Toulouse, France-Ibérie Recherche, 1987.

4. GI 130, trad. fr. Erreurs choisies, 105.

5. Vers 4 : DIESER BAUM WIRD MICH NICHT ÜBERWACHSEN Fischleichen. Anne Ubersfeld note que «le texte de théâtre ne peut jamais être décrypté comme une confidence, ou même l'expression de la "personnalité", des "sentiments" et des "problèmes" de l'auteur ", Lire le Théâtre Editions Sociales, Paris, 1978, p. 22.

6. Sur ce «travail archéologique " (Müller) cf. Müller/Lotringer (1988), Théâtre/Public, n87, p. 6. À propos de sa mise en scène du Briseur de salaire, trente ans après : «Je crois que nous sommes à un point où la fonction de la littérature a à voir avec la libération des morts [...] Je voulais déterrer des choses qui avaient été ensevelies sous la boue, l'histoire, et les mensonges ».

7. GI 133.

8. GI 2,30 .

9. D'abord la nouvelle fiancée doit les reprendre dans son giron («nimm Jason, was du mir gegeben hast / Die Früchte des Verrats aus deinem Samen / Und stopf es deiner Hure in den Schoß ensuite elle les « tue ».

10. Cf. Christian Klein : Heiner Müller ou l'idiot de la République. Le dialogisme à la scène. Peter Lang, 1992, p. 303.

11. "L'Allemagne n'a toujours pas fini déjouer les Nibelungen », entretien avec Heiner Müller réalisé par Urs Jenny et Hellmuth Karasek, Der Spiegel, n¹9, 1983. Trad. fr. in H. Müller, Erreurs choisies, L'Arche, Paris, 1988, p. 106.

12. Erreurs choisies, op. cit., p. 28.

13. H. Müller : «L'effroi, la première apparition du nouveau » (1979), in Erreurs choisies, op. cit., p. 20.

14. M 6, p. 96 («Zerreißung der Fotografie des Autors »).

15. Cf. notre commentaire : Ch. Klein, Heiner Müller ou l'Idiot de la République, op. cit., p. 318-324.

16. Erreurs choisies, op. cit., p. 28.

17. Ibid.

18. Ibid., p. 105.

19. Jacques Derrida, Théorie d'ensemble, Seuil, Paris, 1968, p. 53. 


\section{RÉSUMÉS}

La trilogie de Heiner Müller Rivage à Vabandon Matériau Médée Paysage avec Argonautes (1982) semble d'abord difficile à classer comme texte dramatique. Seuls le contexte et les notes jointes en annexe du texte permettent de le ranger dans le genre théâtral.

Dans Hamlet-machine, une indication scénique prescrivait de « déchirer la photo de l'auteur » et, ce faisant, manifestait ainsi précisément la présence de celui-ci. L'absence de toute didascalie supprime la voix de l'auteur dans le tryptique sur le mythe des Argonautes. Le présent travail interroge le texte lui-même sur les intentions d'une telle dramaturgie-limite. Dans son combat sans compromis pour devenir un sujet, Médée prend elle-même la totalité du spectacle en charge et ses répliques n'ont pas d'interlocuteur réel sur scène : l'assassinat des enfants et de Creuse expérimente au plan verbal, sans la jouer, une phase déterminante de son émancipation. L'annulation symbolique de son rôle d'adjuvante du " héros » épique passe en réalité par la destruction du dialogue théâtral et la décomposition du moi masculin. La cohésion (trompeuse) du récit épique se réduit à l'anecdote et au fragment.

En conséquence, l'absence de didascalies représente non une simple élimination d'indication superflues, mais un élément structurel du texte dramatique.

In Hamletmaschine ließ Müller durch eine Szenenanweisung «das Bild des Autors» auf der Bühne «zerreißen» und machte dadurch dessen Gegenwart manifest. Im Triptychon über den Argonautenmythos (ver) schwindet die Stimme des Autors durch Weglassen der Didaskalien. Die vorliegende Arbeit fragt den Text selbst nach den Intentionen dieser Grenzdramaturgie.

In einem kompromißlosen Kampf ums Subjekt werden führt selbst Medea Regie und ihre Repliken werden an kein Gegenüber auf der Bühne gerichtet: die «Morde» an den Kindern und an Kreuse spielen eine entscheidende Phase der Emanzipation verbal durch aber nicht vor. Die symbolische Zurücknahme ihrer Rolle als Adjuvantin des epischen «Helden» Jason geht eigentlich über die Zerstörung der Dialoghaftigkeit im Theater und über den männlichen Ichzerfall. Epische (Trug-) kohärenz zerfällt ins Anekdotische und Fragmentarische.

Demzufolge ist das Auslassen der Didaskalien mehr als der Verzicht auf überflüssige Anweisungen, es stellt vielmehr ein strukturbildendes Moment des dramatischen Textes dar. 